\begin{tabular}{cc} 
Sharif University of Technology \\
Scientia Iranica \\
SCIENTIA & Transactions B: Mechanical Engineering \\
I RAN I CA & \\
\hline
\end{tabular}

\title{
Numerical study of particulate turbulent flow to investigate recovery period in cleanrooms
}

\author{
A. Pourfarzaneh, A. Jafarian*, and H. Kharinezhad Arani \\ Faculty of Mechanical Engineering, Tarbiat Modares University, Tehran, P.O. Box 14115-143, Iran.
}

Received 15 January 2017; received in revised form 11 June 2017; accepted 16 April 2018

\section{KEYWORDS}

Cleanroom;

Recovery period;

Swirl diffuser;

Computational Fluid

Dynamics (CFD);

Lagrangian approach;

Eulerian approach.

\begin{abstract}
The cleanroom is a controlled space used in various industries such as electronics, and medical and military industries. One of the most important tests to evaluate the performance of the cleanroom is recovery test. Recovery test determines the time period during which a cleanroom returns to its designated cleanliness level after an instant or a period of deliberate or unintentional contamination. In this paper, a thorough investigation of recovery period has been implemented. In the study, air change rate and its pattern were investigated using the Eulerian and Lagrangian approaches as well as LES, DES, and $k-\omega$ SST turbulent models. Simulation results were evaluated through control volume analysis. Parameters such as the air change rate, the number of particles, and pressure and energy consumption in various radial and tangential angles of diffusers were studied. Results showed that radial angle had little positive and occasionally negative effects on recovery period. On the contrary, tangential angle improved decontamination rate; at maximum performance $\left(\beta=45^{\circ}\right)$, it could reduce recovery period by as much as $25 \%$, which in turn reduced energy consumption. In addition, the DES model provided the best and most coinciding answers among all turbulence models.
\end{abstract}

(C) 2019 Sharif University of Technology. All rights reserved.

\section{Introduction}

The recent growth in high-tech industries, especially electronics and medical, has resulted in an increased demand for what is known as a "cleanroom". To put it simply, a cleanroom is an environment within which concentration of aerosols, temperature, and humidity are controlled accurately according to predefined standards.

In a cleanroom, the number of particles larger than a specified size should be less than a specific number per unit of volume (numeric concentration).

\footnotetext{
*. Corresponding author.

E-mail addresses: a.pourfarzaneh@modares.ac.ir ( $A$ Pourfarzaneh); jafarian@modares.ac.ir (A. Jafarian); h.kharinejad@modares.ac.ir (H. Kharinezhad Arani)
}

This specified particle size is between 0.1 and 5 microns. Number of aerosols of any size is determined by the type of activity implemented in the room, and it is called the "Cleanliness Level". Since equipment and personnel in the room are continuously producing and spreading particles, a number of methods are used to maintain this Designated Cleanliness Level (DCL) throughout the room. Introducing aerosol-free air into the room through HEPA filters and creating a pressure difference is a common method of inhibiting particle diffusion from more contaminated areas to the cleaner ones.

Several situations could force the cleanroom to lose its DCL, which could disturb or even halt the production or activity. These situations and the respective recovery scenarios are categorized and shortly discussed below:

- Unsteady flow of particle and air: This scenario 
is mostly associated with Power Outage. When power supply is interrupted, ventilation system stops functioning and the differential pressure between different sections is lost. Due to particle generation, mostly by the personnel, DCL rapidly falls below the designated value. Recovery period is defined as time period during which DCL is reached after restoration of power.

- Unsteady flow of particle and steady airflow: This scenario, also known as Field Test Scenario, mostly occurs when a deliberate contamination is introduced in a specified period of time when there is a steady airflow throughout the room. In this case, recovery is defined as time period during which cleanroom recovers its DCL after contamination process has ceased.

- Steady flow of particle and air: This scenario, also known as Incident Scenario, occurs when a part of the equipment (like a filter or a glove) fails to maintain satisfactory performance and a constant flow of particles is introduced into the airflow. In this scenario, recovery could be defined as the period of time during which a specified fraction of particle generation rate is exhausted through the outlet.

In the absence of a classified definition of recovery period and in order to keep a certain amount of applicability, this research has been implemented based on Power Outage Scenario as it is the most frequent scenario.

Multiple researches have been carried out on cleanrooms, but heretofore, few studies $[1,2]$ have completely and comprehensively investigated duration of recovery and transient removal of contamination in cleanrooms. Lage et al. [2], via a two-dimensional study of contamination removal (Eulerian), have shown that relocation of intake and exhaust vents can improve decontamination rate. In a steady flow study, Mendez et al. [3] considered the effects of intake and exhaust vent configuration in a hospital room. The effect of moving objects on particle distribution in a cleanroom was investigated via Eulerian method by Saidi et al. [4]. In a numerical and experimental study, Chen et al. [5] used the Eulerian method to investigate particle distribution and removal process. Khoo et al. [6] used an experimental study in a steady state flow to inspect rate and level of effective ventilation in particle concentration in a cleanroom. In a numerical analysis, Wang et al. [7] conducted a three-dimensional investigation into both Eulerian and Lagrange methods, using two specified points to compare and inspect turbulent models.

In this paper, a comprehensive investigation has been conducted to predict the recovery time in a cleanroom using Eulerian and Lagrangian approaches. Results have been compared with Control Volume
Analysis as a conventional method. To simulate the flow field, commonly used turbulence models, namely, Large Eddy Simulation (LES), Detached Eddy Simulation (DES), $k-\omega$ SST, and Discrete Random Walk (DRW) model, for the discrete phase were employed. Parameters such as the air change rate and energy consumption in various radial and tangential angles of diffusers were studied.

\section{Case study definition}

The space simulated in this study is a cleanroom of mixed flow type, whose characteristics are accurately described.

This positively pressurized room is part of an industrial complex and consists of two sections. The main part $(3 \mathrm{~m} \times 2 \mathrm{~m} \times 2.5 \mathrm{~m})$ is a unidirectional laminar hood certified as ISO 5 cleanliness class. The second and smaller section ( $3.8 \mathrm{~m}^{2}$ area, $11.4 \mathrm{~m}^{3}$ volume) is certified as ISO 6 cleanliness class and forms the multidirectional conventional flow section.

The nominal air change rate, while unidirectional hood is switched off, is 47 times an hour. The minimum required pressure in the room is $12.5 \mathrm{~Pa}$. Two swirl diffusers $(0.7 \mathrm{~m} \times 0.7 \mathrm{~m})$ supply the air. The exhaust, of rectangular shape $(1.4 \mathrm{~m} \times 0.7 \mathrm{~m})$, is located on one of the side walls. Dimensions, and different parts and section of the room are illustrated in Figure 1.

Before entering the room, the incoming air passes through $\mathrm{H} 13$ class filters with an efficiency of $99.75 \%$.

In order to study the effects of equipment in the recovery period, two different layouts are considered. The first one is as-built layout, which contains a working desk located in the center of the unidirectional hood. This table is $1.5 \mathrm{~m}$ long, $0.6 \mathrm{~m}$ wide, and $0.9 \mathrm{~m}$ high. The second layout, known as at-rest, is exactly the same as as-built mode, but lacks the desk.

According to standards, in order to evaluate cleanliness level of the room, air samples of specified volumes are to be collected from specific spots in the room and particle concentration is to be calculated.

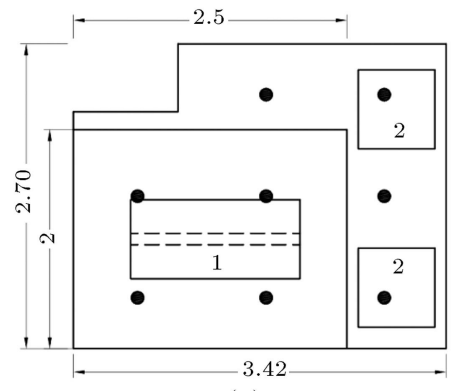

(a)

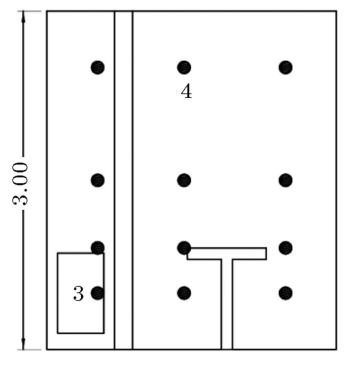

(b)
1: Working desk, 2: Inlet diffuser,

3: Exhaust vent, 4: Sampling unit centroids.

Figure 1. Case study in as-built mode: (a) Plan, and (b) side view. 
Mandatory number and volume of sampling units are calculated from Eqs. (1) and (2), respectively [8].

$$
\begin{aligned}
& N_{s}=\sqrt{A}, \\
& V_{s}=\frac{20}{C_{r}},
\end{aligned}
$$

where $A$ is the area of the room in square meters and $C_{r}$ is the maximum allowable count of the largest particle in DCL. Act of sampling shall be done in the height of the activity uniformly spread throughout the room with a volume not less than 2 liters for each sampling [9].

For a more precise investigation into recovery in our cleanroom, a total number of 32 sample units were considered, 24 units taken in three rows, each of which contained 8 sampling units, uniformly spaced throughout the room in three different heights. The remaining 8 units were located in the height of activity.

In order to be able to use a structured mesh, sampling volumes were not created in the model. Instead, an ASCII format file was exported from Fluent ${ }^{\circledR}$ and used in a Matlab ${ }^{\circledR}$ program to calculate mass concentration in both Lagrangian and Eulerian approaches for each unit. The geometry of these volumes was assumed to be cubic.

To validate our code, its results were compared with a sample Fluent ${ }^{\circledR}$ analysis by creating a sampling volume in the grid. Then, two different reports for average concentration in the sampling unit were extracted from converged solution, the first one with Fluent ${ }^{\circledR}$ itself and the second one through exporting ASCII data and running the Matlab code. Results were exactly the same up to the order of $10^{-4}$.

\section{Control volume analysis}

As the simplest and most comprehensible method, control volume analysis is widely used in order to predict recovery period of a cleanroom. This analysis does not concern airflow pattern or state inside the room, so it can be applied to all three scenarios mentioned before.

The general case for this analysis is shown in Figure 2. Particle instant dispersion is the core assumption of this analysis, which presumes uniform particle concentration throughout the room, including exhaust vent. Applying continuity and mass conservation equations will lead to $[10,11]$ :

$$
\begin{aligned}
\frac{d C(t)}{d t}= & S+\eta_{f} \frac{\dot{m}_{i}}{\rho_{f} V_{f}}\left[\left(1-\frac{\dot{m}_{m}}{\dot{m}_{i}}\right) C_{t}+\frac{\dot{m}_{m}}{\dot{m}_{i}} C_{a}\right] \\
& -\frac{\dot{m}_{i}}{\rho_{f} V_{f}} C(t),
\end{aligned}
$$

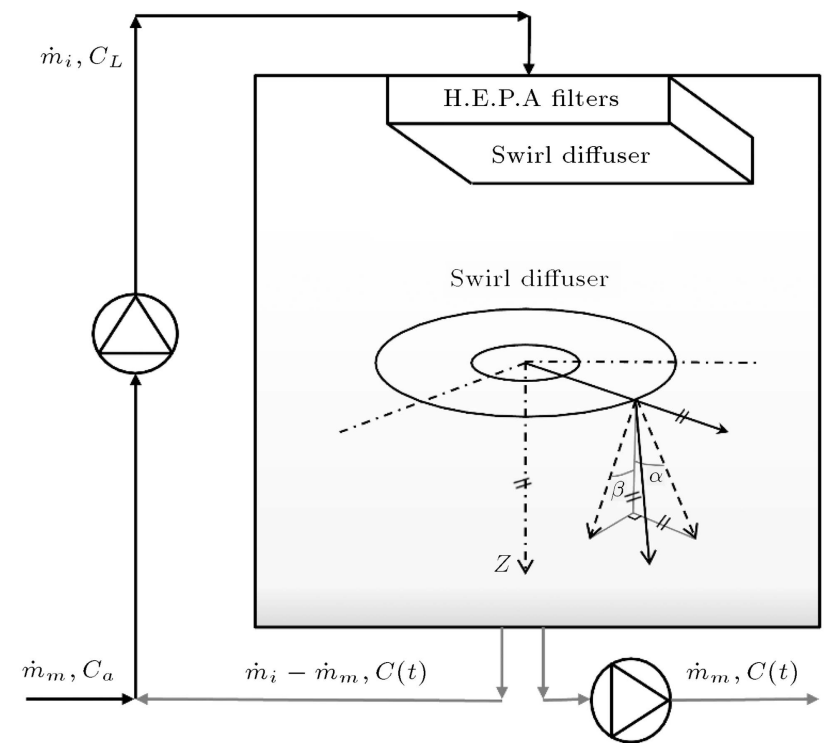

Figure 2. General case for control volume analysis and schematic of diffuser flow angles.

$$
\begin{aligned}
C(0)= & C_{0}, \quad A C H=\frac{\dot{m}_{i}}{\rho_{f} V_{f}}, \\
C(t)= & C_{0} e^{-A C H \cdot \xi t}+\frac{A C H \cdot F \cdot C_{a}\left(1-\eta_{f}\right)+S}{A C H \cdot \xi} \\
& \left(1-e^{-A C H \cdot \xi t}\right) .
\end{aligned}
$$

In this equation, $\xi$ is:

$$
\xi=1-\left[(1-F) \times\left(1-\eta_{f}\right)\right] .
$$

$F$ is make-up air fraction to total recirculated mass flow of air and is defined as:

$$
F=\frac{\dot{m}_{m}}{\dot{m}_{i}} .
$$

The first and second parts of Eq. (4) are known as decontamination and contamination parts, respectively. If recovery, defined as the time period required for particle concentration, decreases by two orders of magnitude, we will have:

Solving the equation will result in:

$$
\begin{aligned}
t_{0.01}= & -\frac{1}{A C H \cdot \xi} \ln \left[\left(\frac{C_{0}}{100}-\frac{A C H \cdot F \cdot C_{a}\left(1-\eta_{f}\right)+S}{A C H \cdot \xi}\right)\right. \\
& \left.\times\left(\frac{A C H . \xi}{A C H . \xi C_{0}-A C H \cdot F \cdot C_{a}\left(1-\eta_{f}\right)+S} \xi\right)\right] \cdot(7)
\end{aligned}
$$

Supposing 100\% efficiency for HEPA filters, recovery time is reduced to:

$$
t_{0.01}=-\frac{1}{A C H} \ln \left[\left(\frac{C_{0}}{100}-\frac{S}{A C H}\right) \times\left(\frac{A C H}{A C H \cdot C_{0}+S}\right)\right]_{(8)} .
$$

This equation indicates that when HEPA filters per- 
formance is $100 \%$, recovery period is independent of recirculated and make-up air fractions.

Several protocols for cleanrooms, e.g., O\&M, explicitly order evacuation of the cleanroom in case of power failure, while others associate it with power outage duration. In our case study, due to the absence of emergency or uninterruptable power supplies, its protocol stresses evacuation in case of a power failure. Therefore, particle source term is eliminated and Eq. (9) will merge into:

$$
t_{0.01}=\frac{16578.6}{A C H}
$$

Also, fractional concentration profile equation will be:

$$
\frac{C(t)}{C_{0}}=e^{-A C H . t} .
$$

Simplicity of the volume control analysis is its greatest strength and weakness at the same time; although it makes analysis easier, in addition to the possibility of deviation from real case, the effects of parameters other than air change rate are not considered.

\subsection{Assumptions and equations}

The first governing equation of fluid's dynamics is continuity. Regarding the limit of air velocity to amounts much lower than the speed of sound, the incompressibility assumption is valid and, after averaging, the continuity equation is simplified to Eq. (11) [12]:

$$
\nabla \cdot \overline{\mathbf{u}}=\mathbf{0} .
$$

Solving the continuity equation is not meaningful on its own. Therefore, it will be enforced through correction of pressure field. In this study, SIPMLE correction with a first-order upwind scheme has been utilized to modify pressure filed in each time step.

The second most important equation governing fluid's dynamics is the linear momentum equation, also known as the "Navier-Stokes" equation. Considering the stream incompressible, after averaging, the equation is simplified into Eq. (12):

$$
\begin{aligned}
\rho_{f}\left(\frac{\partial \bar{u}}{\partial}\right. & \left.+\bar{u} \frac{\partial \bar{u}}{\partial x}+\bar{v} \frac{\partial \bar{u}}{\partial y}+\bar{w} \frac{\partial \bar{u}}{\partial z}\right) \\
& =\left(\rho_{f} g_{x}-\frac{\partial \bar{P}}{\partial x}+\mu_{f}\left(\frac{\partial^{2} \bar{u}}{\partial x^{2}}\right.\right. \\
& \left.\left.+\frac{\partial^{2} \bar{u}}{\partial y^{2}}+\frac{\partial^{2} \bar{u}}{\partial z^{2}}\right)\right)+\frac{\partial}{\partial x}\left(-\rho_{f} \overline{u^{\prime} u^{\prime}}\right) \\
& +\frac{\partial}{\partial y}\left(-\rho_{f} \overline{u^{\prime} v^{\prime}}\right)+\frac{\partial}{\partial z}\left(-\rho_{f} \overline{u^{\prime} w^{\prime}}\right)
\end{aligned}
$$

Similarly, it can be done for the other two directions.
Regarding the absence of spillage as the boundary condition, all the components of velocity on the walls are equal to zero:

$$
\bar{u}=0, \quad \bar{v}=0, \quad \bar{w}=0 .
$$

\subsection{Turbulent flow equations}

To replace averaged product of two fluctuating terms in Eq. (12) (known as Reynolds stress), Boussinesq approximation is used, which assumes isotropic turbulent filed. Reynolds stresses can be approximated to mean velocity as in Eq. (14). For this, Boussinesq approximation is used:

$$
\begin{aligned}
\left(-\rho_{f} \overline{u_{l}^{\prime} u_{J}^{\prime}}\right)= & \mu_{t}\left(\frac{\partial \bar{u}_{i}}{\partial x_{j}}+\frac{\partial \bar{u}_{j}}{\partial x_{i}}\right) \\
& -\frac{2}{3}\left(\rho_{f} k \delta_{i j}+\mu_{t} \frac{\partial \bar{u}_{k}}{\partial x_{k}}\right) .
\end{aligned}
$$

With substitution in Eq. (12), we arrive at Eq. (15):

$$
\begin{aligned}
& \rho_{f}\left(\frac{\partial \bar{u}}{\partial t}+\bar{u} \frac{\partial \bar{u}}{\partial x}+\bar{v} \frac{\partial \bar{u}}{\partial y}+\bar{w} \frac{\partial \bar{u}}{\partial z}\right) \\
& \quad=\left(\rho_{f} g_{x}-\frac{\partial \bar{P}}{\partial x}+\mu_{e f f}\left(\frac{\partial^{2} \bar{u}}{\partial x^{2}}+\frac{\partial^{2} \bar{u}}{\partial y^{2}}+\frac{\partial^{2} \bar{u}}{\partial z^{2}}\right)\right),
\end{aligned}
$$

where $\mu_{e f f}$ is the effective diffusion coefficient.

So far, several studies have been conducted on different methods of turbulent flows modelling. Ruaud et al. [13], by comparing $k-\varepsilon$ and $k-\varepsilon$ RNG models, concluded that the latter was more accurate for simulating particle motion. Zhang and Chen [14] used $k-\varepsilon$ model in their study with an acceptable accuracy, although the model demonstrated deviation from experimental results in several situations. In a similar study, Wang et al. [7] used $k-\varepsilon$ RNG, LES (Lilly-Smagorinsky sub-grid), and DES models and deduced that RANS/URANS methods could not predict correct particle concentration, but the other two methods presented more suitable results.

In this study, LES turbulence with LillySmagorinsky sub-grid scale, DES with $k-\omega$ SST subgrid scale, and $k-\omega$ SST as URNAS method are used. In the following, the aforementioned models are explained:

- $k-\omega$ SST: This method is based on transport equations of turbulent kinetic energy and specific dissipation rate of turbulent energy. Both $k-\omega$ and modified $k-\varepsilon$ methods are combined, which allows $k-\omega$ method to be used in regions close to walls and $k-\varepsilon$ to be applied to areas far from it [15]. This method uses transversal dispersion in $\omega$ equation; also, the definition of turbulent viscosity is changed to include turbulent tension transfer.

These characteristics help this method to be 
widely used in problems ranging from flows with adverse pressure gradients to airfoils and shock waves. $K$ and $\omega$ equations are illustrated in Eqs. (16) and (17):

$$
\begin{aligned}
\frac{\partial\left(\rho_{f} k\right)}{\partial t} & +\frac{\partial\left(\rho_{f} \bar{u}_{i} k\right)}{\partial x_{i}}=\frac{\partial}{\partial x_{i}}\left[\Gamma_{k} \frac{\partial k}{\partial x_{i}}\right] \\
& +\tilde{G}_{k}-Y_{k}+S_{k} \\
\frac{\partial\left(\rho_{f} \omega\right)}{\partial t} & +\frac{\partial\left(\rho_{f} \bar{u} \omega\right)}{\partial x_{i}}=\frac{\partial}{\partial x_{i}}\left[\Gamma_{\omega} \frac{\partial \omega}{\partial x_{i}}\right]+\tilde{G}_{\omega}-Y_{\omega} \\
& +S_{k}+D_{\omega}
\end{aligned}
$$

where $G, Y$, and $S$ represent production, dissipation, and source terms, respectively. Also, $D_{\omega}$ is crossdiffusion $[16,17]$.

- LES: One of the most widely used models of turbulent flow is large eddy scale. In LES method, unlike the DNS method, in which the entire field is solved accurately, large structures of the flow field are calculated directly. Using LES allows for larger time steps and coarser grid than using DNS does, although both of these quantities are still smaller than URNAS methods. In this method, subgrid filters omit flow field scales smaller than a specific time and length. This filter appears in Eq. (18) [18]:

$$
\tilde{\phi}(x, t)=\int \phi(\mathbf{r}, t) G(\mathbf{x}-\mathbf{r}, t) d \mathbf{r}
$$

where $G$ is the convolution kernel of the chosen filter. The averaged equation in $X$ direction will be as Eq. (19):

$$
\begin{aligned}
\frac{\partial \tilde{u}_{l}}{\partial t} & +\frac{\partial \tilde{u}_{l} \tilde{u}_{J}}{\partial x_{j}}=\left(\tilde{g_{x}}-\frac{1}{\rho_{f}} \frac{\partial \tilde{P}}{\partial x_{i}}+\frac{\partial}{\partial x_{j}}\left(v \frac{\partial u_{i}}{\partial x_{j}}\right)\right) \\
& -\frac{1}{\rho_{f}} \frac{\partial}{\partial x_{j}}\left(\tau_{S G S}\right)_{j i} .
\end{aligned}
$$

In this research, Smagorinsky subgrid model has been used. Therefore, turbulent stress term is defined as Eq. (20):

$$
\left(\tau_{S G S}\right)_{j i}=2\left(v+v_{s}\right) \bar{S}_{i j}
$$

where $\bar{S}_{i j}$ is the rate of strain and is calculated from Eq. (21):

$$
\bar{S}_{i j}=\frac{1}{2}\left(\frac{\partial \bar{u}_{i}}{\partial x_{j}}+\frac{\partial \bar{u}_{j}}{\partial x_{i}}\right) .
$$

The turbulent viscosity is calculated from Eq. (22):

$$
v_{s}=L_{s}^{2}\left(\bar{S}_{i j} \bar{S}_{i j}\right)^{\frac{1}{2}}
$$

The $L_{s}$, the mixing length for subgrid in threedimensional flow, is calculated from Eq. (23):

$$
L_{s}=C_{0}(\Delta x \Delta y \Delta z)^{\frac{1}{3}}
$$

where $C_{0}$ is a coefficient with the value between 0.094 and 0.2 . This coefficient is constant throughout the solution.

- DES: Also known as hybrid LES/RANS method, it was created for internal flows with high Reynolds numbers. Using LES around the walls in these types of flow fields increases the computing cost. In fact, the only difference between this method and LES is the use of RANS in boundary layers. For calculation of turbulent viscosity, LES equations should be used. But, in the $K-\omega$ SST based DES method, in the boundary layers, the turbulent kinetic energy expression $(K-\omega \mathrm{SST})$ is corrected [19]:

$$
\begin{aligned}
& Y_{k}=\rho_{f} \beta^{*} k \omega F_{D E S}, \\
& F_{D E S}=\max \left(\frac{L_{t}}{C_{D E S} \Delta_{\max }}, 1\right),
\end{aligned}
$$

where $C_{D E S}$ is a calibration coefficient with the value of 0.65 and $\Delta_{\max }$ is the maximum size of the local grid.

\subsection{Lagrangian approach}

Since a particle is a discrete phase, its equations should be analyzed separately in a reference coordinate system. Particle's equation of motion is Newton's second law.

The forces acting on the particle are drag, gravity, and buoyancy forces. They are the only effective forces in the present work. Therefore, the final particle's equation of motion is transformed $[7,20]$ into the following form in Eq. (26):

$$
\begin{aligned}
& \frac{d \mathbf{u}_{p}}{d t}=\frac{1}{\tau_{r}}\left(\mathbf{u}_{f}-\mathbf{u}_{p}\right)+\mathbf{g}\left(1-\frac{\rho_{f}}{\rho_{p}}\right) \\
& \tau_{r}=\frac{\rho_{p} d_{p}^{2} C_{c}}{18 \mu_{f}} .
\end{aligned}
$$

\subsection{Effects of turbulent field on particles}

The discrete random walk method is used to apply the effects of turbulence field on particle's equation of motion. In this method, the turbulence is assumed isotropic; as a result, its three components are equal. Therefore, velocity of fluid in the equation of motion is formed as Eq. (28) demonstrates [21]:

$$
\begin{aligned}
\mathbf{u}_{f}= & \left(\bar{u}+\zeta \sqrt{\frac{2 k}{3}}\right) \hat{l}+\left(\bar{v}+\zeta \sqrt{\frac{2 k}{3}}\right) \hat{J} \\
& +\left(\bar{w}+\zeta \sqrt{\frac{2 k}{3}}\right) \hat{k}
\end{aligned}
$$


where $k$ is turbulence kinetic energy and $\zeta$ is a random number of Gaussian distribution with zero mean value and standard deviation of 1 . During the analysis, the discussed random number would change in accordance with the turbulence field.

In this analysis, both large and small turbulent length scales are compared with particle radius. Since neither of the large nor small scales in the whole field are smaller than particle radius in the severest turbulence, the effects of particles on turbulent field are considered to be negligible.

In order to create a realistic field for Lagrangian approach (as the initial condition), first, a unidirectional velocity field is defined in the entire solution geometry with a zero gravity field and zero turbulent kinetic energy. Then, particles are released into the room, forming a uniform but not randomly distributed field of particles. Afterwards, the particles are allowed to be dispersed in a zero-velocity gravity field with turbulent kinetic energy of dissipation rate 1 ; therefore, a physical and realistic field will be formed, which is randomly uniform. At this point, the decontamination process is initiated (ventilation system starts up) to reach a point with number of particles decreased to $1 / 200$ to $1 / 150$ of the initial count.

\subsection{Eulerian approach}

Mass transfer equations are used in Eulerian approach. By applying the transport equation for each species, Eq. (29) is achieved [22]:

$$
\frac{\partial C}{\partial t}+u \frac{\partial C}{\partial x}+v \frac{\partial C}{\partial y}+w \frac{\partial C}{\partial z}=\nabla(\nabla \cdot J)+S
$$

where $C$ is the concentration (mass fraction) of the intended species and $J$ is its flux vector, which can be obtained from Fick's law:

$$
J_{i}=-D \frac{\partial C}{\partial x_{i}},
$$

where $D$ is Fick's coefficient or the diffusion coefficient for the intended particle. Through averaging and Boussinesq approximation, Eq. (29) will change into:

$$
\begin{aligned}
& \frac{\partial \bar{C}}{\partial t}+u_{i} \frac{\partial \bar{C}}{\partial x_{i}}=\left(D+\Gamma_{t}\right)\left(\frac{\partial^{2} \bar{C}}{\partial x_{i} \partial x_{i}}\right), \\
& \Gamma_{t}=\frac{\mu_{t}}{S c_{t}},
\end{aligned}
$$

where $S c_{t}$ is turbulent Schmidt number, which must be considered 0.7 to achieve realistic solutions [23].

Mixture of air and carbon monoxide is used for Eulerian analysis to minimize change of carrier fluid's characteristics due to similarity of carbon monoxide's properties (molecular mass, density, and viscosity) to those of air.

\subsection{Conformity analysis}

In order to quantify how close numerical results are to the results of experiments, correlation factor according to Pearson's R-squared method is applied. If $X$ and $Y$ are two vectors of the same size (e.g., data of numerical analysis and experimentation obtained in the specified intervals of time), then Pearson's correlation factor is defined as Eq. (33):

$$
C F=\frac{\operatorname{Cov}(X, Y)}{\Psi_{X} \Psi_{Y}}
$$

Numerator is covariance of the given vectors and defined as:

$$
\operatorname{Cov}(X, Y)=\frac{\sum_{i=1}^{m}\left(x_{i}-\bar{x}\right)\left(y_{i}-\bar{y}\right)}{n-1} .
$$

Denominator of Eq. (33) is product of each vector's standard deviation and for vector $X$ is defined as:

$$
\Psi_{X}=\sqrt{\frac{\sum_{i=1}^{m}\left(x_{i}-\bar{x}\right)^{2}}{n-1}} .
$$

\subsection{Model validation}

Before starting the analysis of our studied case, it is necessary to verify the chosen models. The experimental results of $\mathrm{Lu}$ et al. [24] were used for this goal. The space used in their study included two similar enclosures, each $2.5 \mathrm{~m}$ in length, $3 \mathrm{~m}$ in width, and $2.4 \mathrm{~m}$ in height, joined together with a sliding door of $0.9 \mathrm{~m}$ height and $0.7 \mathrm{~m}$ width. Both air diffusers were $1 \mathrm{~m}$ wide and $0.5 \mathrm{~m}$ high, but located in different levels and sections of the room. It is worth noting that the results of this experimental study were obtained for the experiments of Wang et al. [7].

The particles used in this experiment have diameters between 0.5 and 5 microns. At first, the sliding door between the two sections is closed, while the particles are released into first section of the room to reach a uniform dispersion. Then, ventilation devices with air change rate of 10.26 start working while the sliding door opens at the same time. Particle concentrations reading in each section is done in $1 \mathrm{~min}$ intervals.

\subsubsection{Turbulent models}

In this study, 3 turbulent models of LES, DES, and URANS were investigated. Regarding the use of swirl diffusers and presence of high-speed flow fields, and high curvature of the stream, this model benefited from $K-\omega$ SST method. $K-\omega$ SST was also used in boundary layers of the DES method, while Lily-Smagorinsky sub-grid model was employed in LES.

Results show that although $k-\omega$ SST predictions are not entirely consistent with experiments in both 


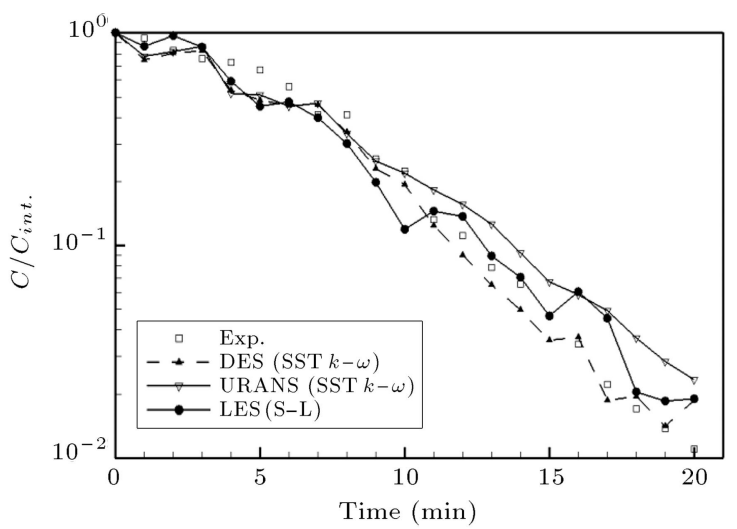

(a)

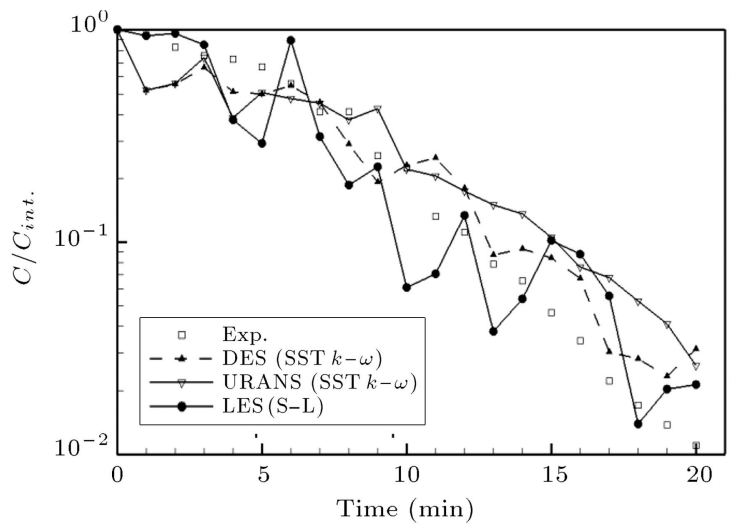

(b)

Figure 3. Comparison of numerical and experimental analysis results for turbulent models: (a) Eulerian approach and (b) Lagrangian approach.

Eulerian and Lagrangian methods, compared with $k-\varepsilon$ RNG results (used in the previous studies), they have improved significantly. Besides, although LES and DES methods predict concentration variations better in the Lagrangian method, Eulerian method provides better overall results. Figure 3 shows a comparison between both numerical Eulerian and Lagrangian methods.

By comparing correlation coefficients obtained from the results of these three methods with experimental values in both sections and both approaches, it is apparent that DES method provides the best and most accurate results.

\subsubsection{Particles boundary conditions}

Studying the effects of boundary conditions on the results showed that trap boundary condition did not provide correct results. In reflect boundary condition, results have little dependency on coefficient of restitution, although results obtained with coefficient of restitution of 1 present a greater correlation with experimental and Eulerian approach results.

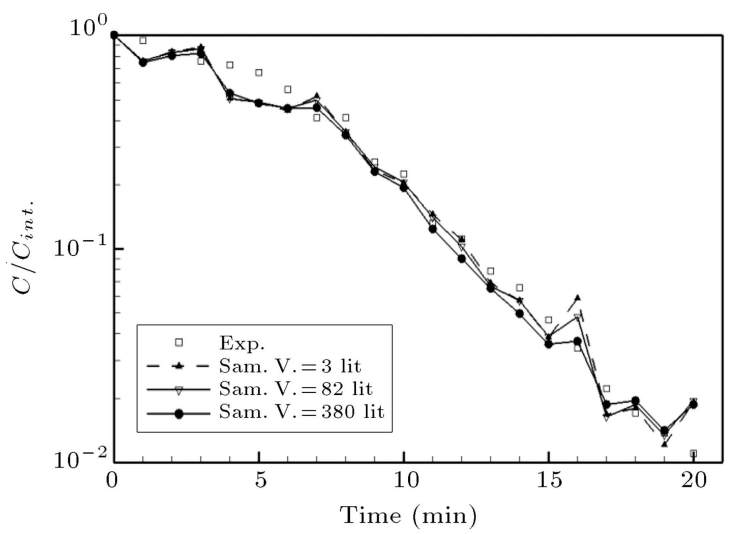

(a)

\subsubsection{Sampling unit volume}

As described in Section 2, sampling volumes are not formed inside our grid and mean concentrations are calculated inside a Matlab code. Therefore, it is highly likely for sampling units to have closely but not exactly the same volumes. Thus, it is required to perform a sensitivity analysis on sampling unit volumes. To measure the effects of change in the volume of sampling unit on the results extracted from DES model by unity coefficient of restitution, 3 different volumes in cubic shape were considered. The results, as presented in Figure 4, showed that in the Eulerian analysis, the outcome was not very dependent on sampling unit size; however, with increasing volume, it converged on a specific amount. On the contrary, volume in the Lagrangian approach has a more pronounced effect on results. Although little difference was observed between 82 and 340-liter volumes, when volume decreased to 3 liters, the results showed a noticeable fluctuation, which was due to sampling unit size being comparable to mean particle distance. Thus, as long as the sampling unit volume is not comparable with the room

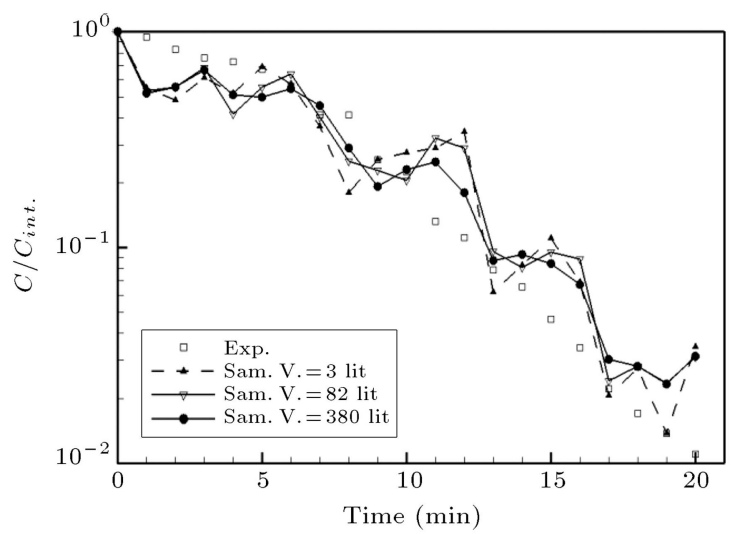

(b)

Figure 4. Comparison of numerical and experimental analysis results for sampling unit volume: (a) Eulerian approach and (b) Lagrangian approach. 
volume and mean particle distance is incomparable to sampling unit characteristic length, the result is more dependent on the location of the sampling unit rather than on its size.

\section{Study of grid, time step, and number of particles}

After the models and boundary conditions are investigated, a proper grid independency to the results should be obtained. In addition, similar procedure should be carried out for time step. Grid and time step independency is studied simultaneously in this research, that is, since grid independency is not achieved with large time steps, in smaller time steps, all the studied grids over the 24 sampling volumes are graphed over time and compared in their basic analysis and concentration reduction process mode. The grids studied had 15457 , 25920, 62080, 122500, 207360, and 496640 cells. Time steps also started with 0.2 seconds and were halved in 4 stages, reaching 0.01 in the fifth stage. Results show that 0.025 second is suitable time step and no perceivable change is observed in results by changing the grid from 207360 to 496640 . Therefore, grid number of 207360 and time step of 0.02 second are chosen for analysis.

Calculation cost of Lagrangian analysis is directly proportional to the number of particles present in the field of solution. Minimum number of particles for reaching an independent solution is highly dependent on the number of computational cells in the field. Previously, in other researches, proper ratio of particle count to grid cells for achieving this independency was stated. According to previous studies, this ratio is regarded as 0.5 and the results are compared by increasing the number of particles (107246, 165240, 364715 , and 563547, respectively). The results show that by increasing the number of particles, the analysis results converge on a specific value, so that with increase from 364715 to 563547 , no significant change occurs. Therefore, the aforementioned ratio is approximately determined to be 1.8 .

\subsection{Investigated parameters}

To study recovery time in the intended case study, three parameters are changed. These three parameters are ventilation rate of the room $(\mathrm{ACH})$, tangential entry angle $(\alpha)$, and radial entry angle $(\beta)$. Both tangential and radial angles are separately set to $15,30,45,60$, and 75 degrees.

\section{The results of the studied room}

\subsection{Control volume interpretation}

One of the aspects concerned in this research is the deviation of control volume analysis from real results, described in Section 2. In the first encounter, instead of observing each sampling unit, it is preferred to study the behavior of the case study as a whole. Therefore, we performed a control volume interpretation, which made it possible to compare results in large scale with control volume analysis.

For each parameter of study, namely, air change rate per hour, radial angle, and tangential angle on the inlet, fractional particle count plotted against time in a semi-logarithmic scale is shown in Figure 5. On all pictures, control volume analyses are also plotted in order to perform a better comparison. Intersection of each curve with horizontal axis indicates recovery period.

The first picture indicates that in case of increase in air change rate, recovery period will decrease as expected, although it does not demonstrate much deviation from control volume analysis. This deviation even decreases to zero when air change is doubled.

In the middle picture, when increasing radial angle of inlet flow to $45^{\circ}$, recovery period will increase by $12 \%$; but, it will decrease and even reach the same amount as that of vertical flow when $\alpha=75^{\circ}$. Although changing radial angle may have effects on recovery period, it will not help the room to recover faster than control volume analysis.

As for the third picture, increasing swirl angle of the inlet flow to $45^{\circ}$ decreases recovery period experience by $28 \%$, although it increases once more when $\beta=75^{\circ}$. Unlike radial angle, which has negligible effect, tangential angle not only causes noticeable drop of recovery period, but will also make the whole system to recover faster than control volume analysis.

\subsection{Change in pressure and energy consumption}

Since pressurizing is one of the necessary factors of cleanroom design, change in pressure in coordination with other parameters is of utmost importance. On the other hand, reducing energy consumption is an important requirement of today's industry. Therefore, change in pressure and energy consumption is investigated in this section.

In most modern cleanrooms, adjustable outlet vents are used to create minimum required pressure; these diffusers are opened only enough to create required pressure in the room.

In order to consider the required pressure and energy, the pressure at room inlet diffusers and mean pressure at room entrance are calculated in every particle concentration reading. Mean pressure until recovery and the energy requirements are calculated by control volume criterion [Eq. (36)]:

$$
W=\left(\bar{P}_{\text {in }}-\bar{P}_{\text {out }}\right) V_{a} .
$$

Energy and pressure ratio are obtained through 


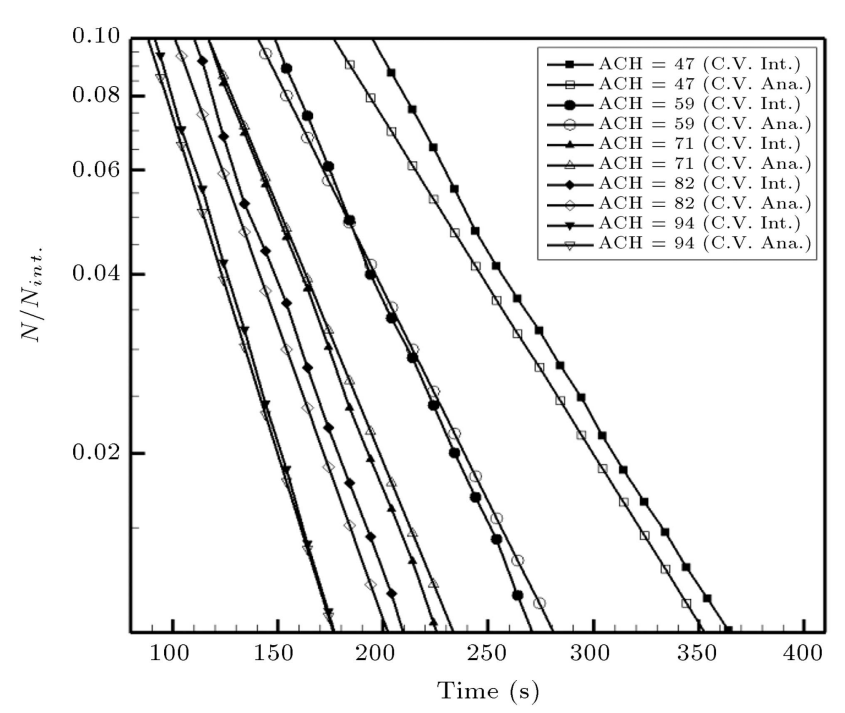

(a)

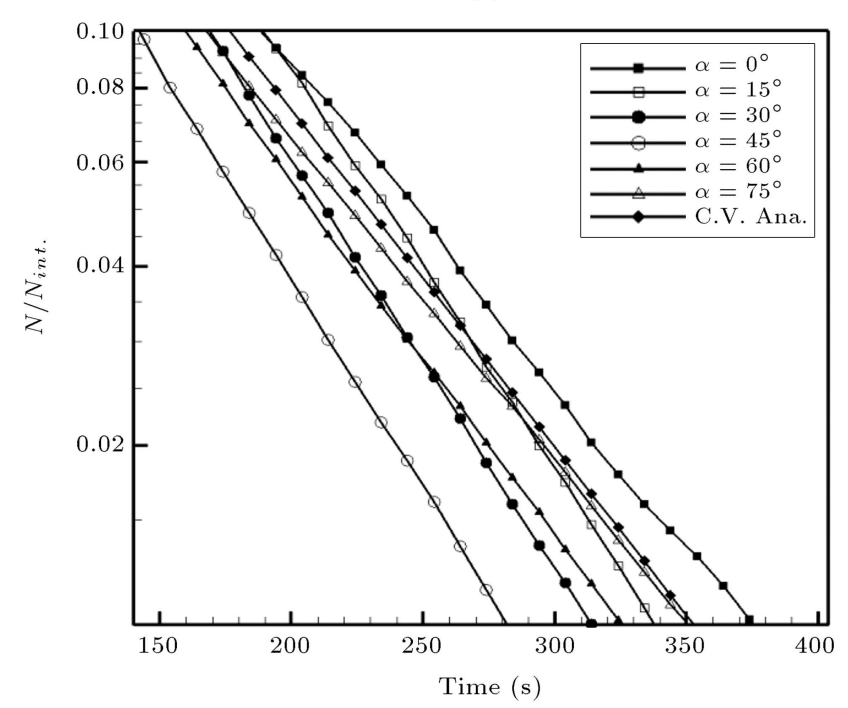

(b)

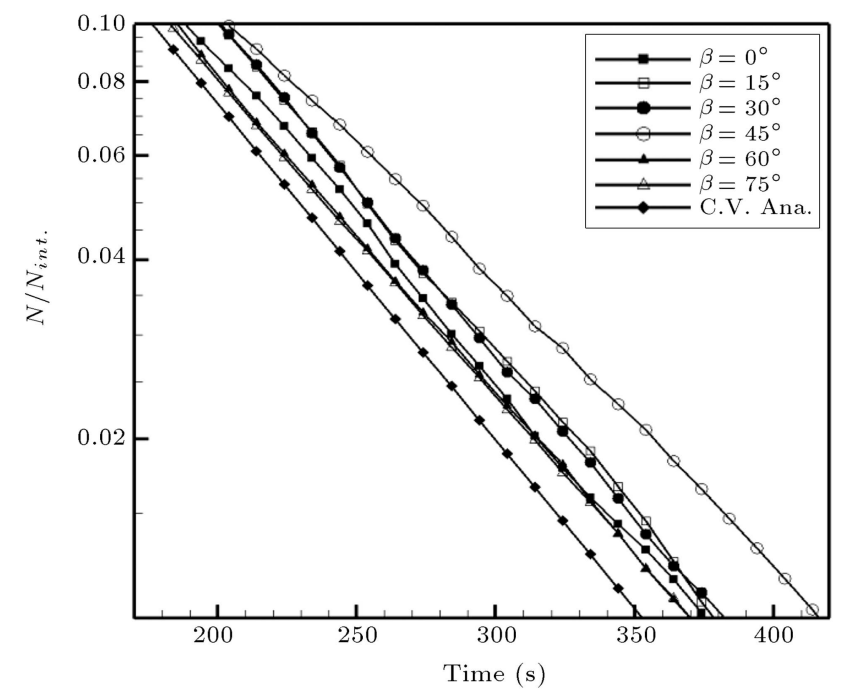

(c)

Figure 5. Control volume interpretation results against ventilation rate (a), radial angle (b), and tangential angle (c) and comparison with control volume analysis.
Eqs. (37) and (38). Since exhaust pressure equals zero, and air volume is equal to intake flow multiplied by recovery time, we arrive:

$$
\begin{aligned}
& \text { Energy ratio }=\frac{W}{W_{\text {ref. }}}, \\
& \text { Pressure ratio }=\frac{\bar{P}_{\text {in }}-\bar{P}_{\text {out }}}{\left.\bar{P}_{\text {in }}\right|_{\text {ref. }}-\left.\bar{P}_{\text {out }}\right|_{\text {ref. }} .} .
\end{aligned}
$$

The subscript (ref.) refers to value of each quantity in reference state, i.e., air change rate at 47 and both radial and tangential angles at zero. Results of both quantities are illustrated in Figure 6.

Results clearly indicate that pressure ratio follows a quadratic pattern with change in ventilation rate, which is physically acceptable. Unlike air change rate, change in both radial and tangential inlet flows does not have a noticeable effect on pressure ratio, although it might slightly weaken pressure field inside the room.

Like pressure ratio, energy ratio relatively follows the same quadratic dependence with change in ventilation rate, that is, increasing ventilation rate by $n$ times roughly increases fan filter unit absorbed power by $n^{3}$ times, while reducing the recovery time by about $n$ times. Although air change rate might have a direct and noticeable effect on consumed energy, it does not happen with radial and tangential angles. As change in pressure is negligible and air change is kept constant at 47 , energy is mainly influenced by a change in recovery time.

\subsection{Sampling units analysis}

In the previous section, only a control volume approach was considered to monitor recovery period, pressure, and energy consumption. Although it gave an overall perspective of what happened in cleanroom when changing ventilation parameters, it did not specify whether cleanroom had recovered based on all sampling units. Therefore, in this section, and as a more precise analysis, the behavior of sampling units is investigated to verify cleanroom recovery.

To this point, results have definitely indicated that, when considering the whole room, decontamination process does not coincide with control volume analysis closely, but still follows a control volume pattern, creating a straight line on semi-logarithmic diagram against time. On the other hand, the data acquired through time for all sampling units and all rates of air change or inlet flow pattern is too vast; thus, we need a proper and compact way to present and interpret results. Therefore, in order to prevent observing each sampling unit during recovery, their behavior is fitted to a function. Since the behavior of the room largely corresponds to control volume analysis, the exponential function of control volume analysis is considered. The fitting, as seen in Eq. (39), 


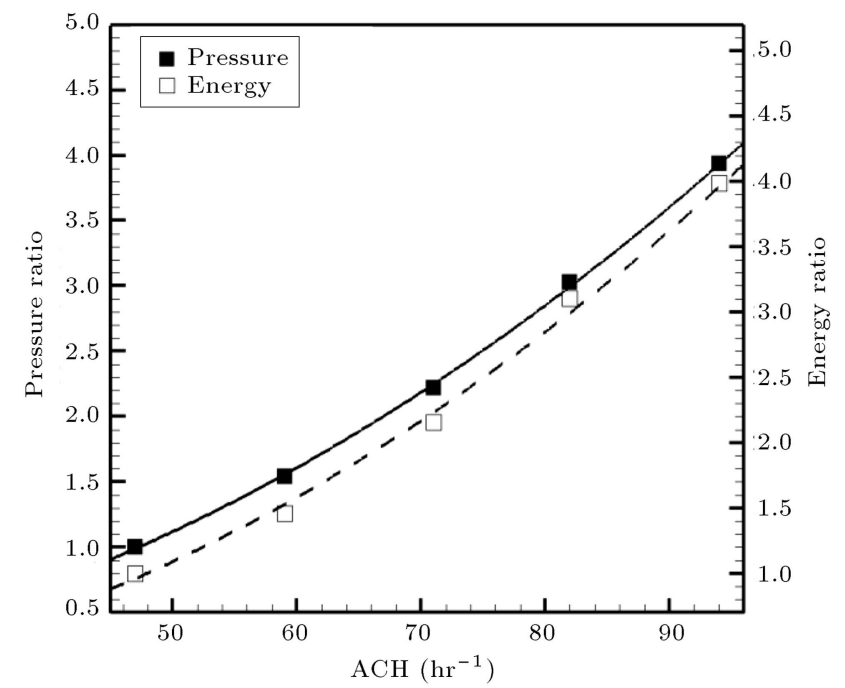

(a)

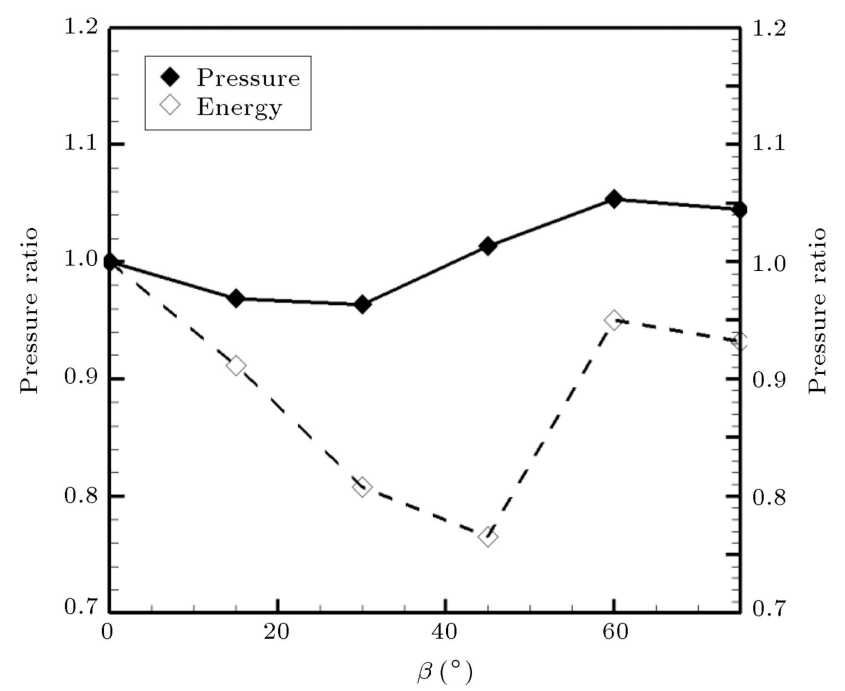

(b)

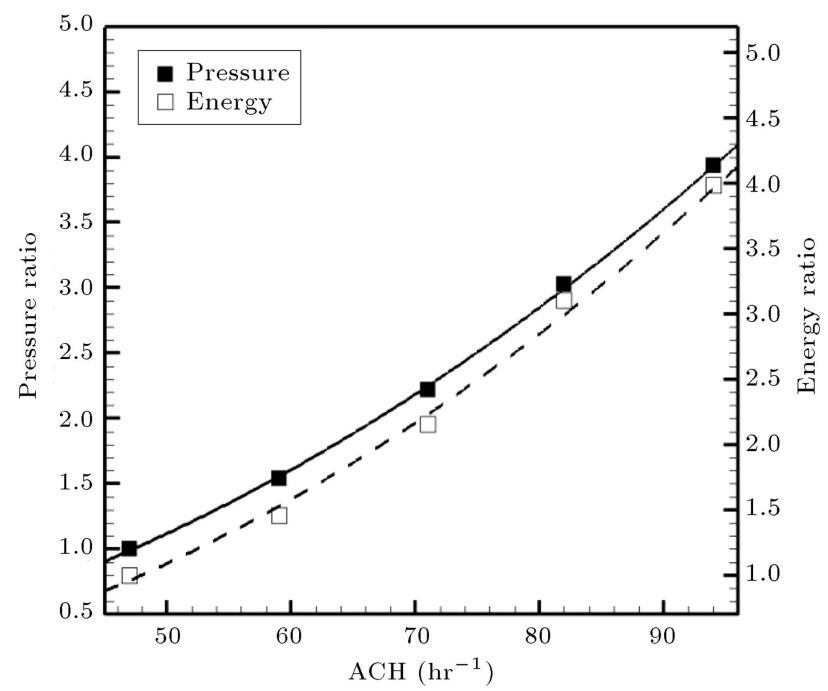

(c)

Figure 6. Change in pressure ratio and consumed energy ratio for recovery against air change rate (a), radial angle (b), and tangential angle (c). will give the best results:

$$
\frac{C_{S U}}{C_{0}}=e^{-\frac{a(t-b)}{3600}}
$$

which means the behavior of sampling volume is regarded as a control volume with a ventilation rate of $a$, but a delay or early offset time of $b$ is considered to compensate for the probable deviation.

\subsection{Recovery performance and recovery period}

The recovery period in each sampling unit is the required time for fractional concentration to reach 0.01. At first glance, obtaining recovery period for each sampling unit necessitates observation of unit's behavior in a specified interval of time; but, calculating the recovery period by this method is not possible because of the following reasons.

First, collected data is discrete and calculating accurate recovery period time requires suitable interpolation and extra mathematical operations.

Second, recovery is indistinguishable, that is, in some sampling units, especially in Lagrangian approach, fractional particle concentration may exceed 0.01 long after it has recovered. Although these volumes may recover in a short while, this behavior makes exact instant of recovery indefinite.

The amount of data in this study is quite high and, therefore, practical calculation of recovery time by this method for all sampling units with each approach, geometry, and parameter is time consuming.

Thus, recovery time can be calculated after fitting a proper function to the results of each sampling unit. For assessing the recovery time in each unit, the left side of Eq. (38) is equal to 0.01 and it is solved for $t$, leading to Eq. (40), which presents recovery time in seconds:

$$
t_{\text {rec. }}=\frac{16578.6}{a}+b .
$$

In order to compare sampling unit recovery with control volume analysis, recovery performance is defined through Eq. (41):

$$
\eta_{V}=\frac{a}{A C H}
$$

This equation simply indicates whether a sampling unit overtakes or falls behind control volume analysis. If recovery performance is greater than 1 , it means that sampling unit recovery is faster than what CVA has predicted; the opposite is also true if recovery performance is less than 1 .

\subsection{Mean and variance of recovery performance}

Mean value and variance of recovery performance among 32 sampling units are calculated for both 

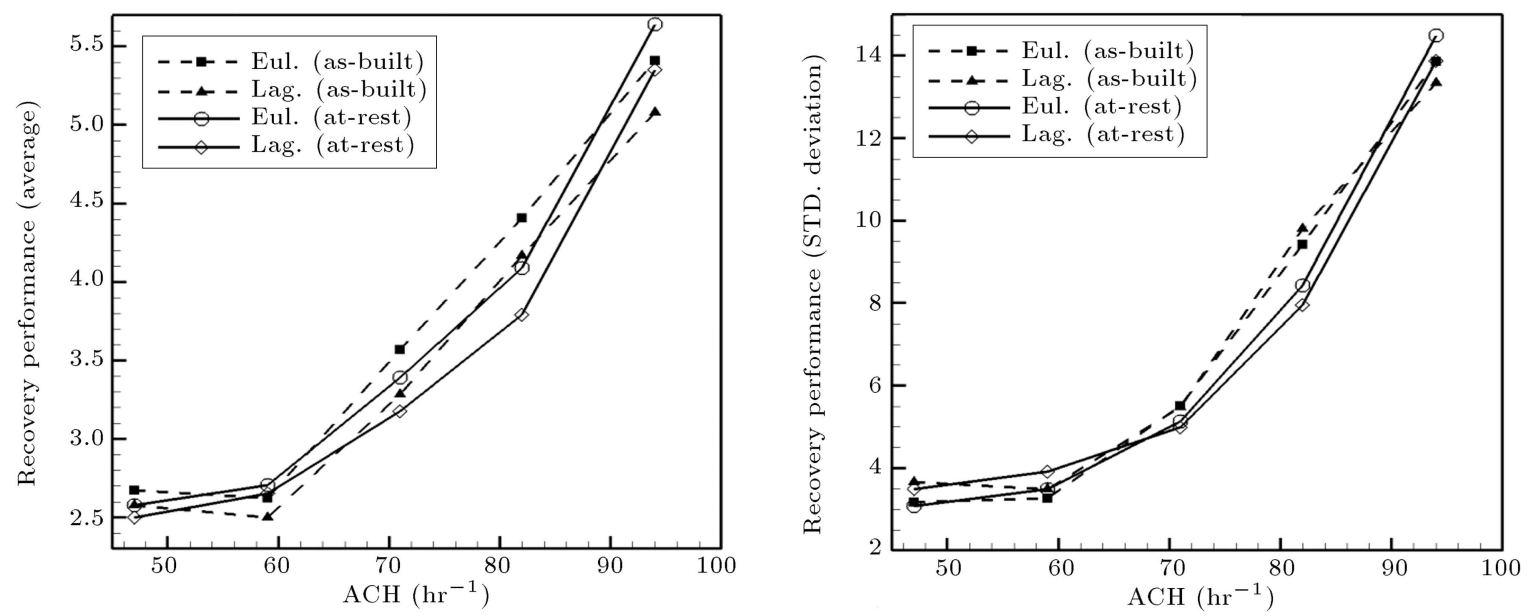

(a)
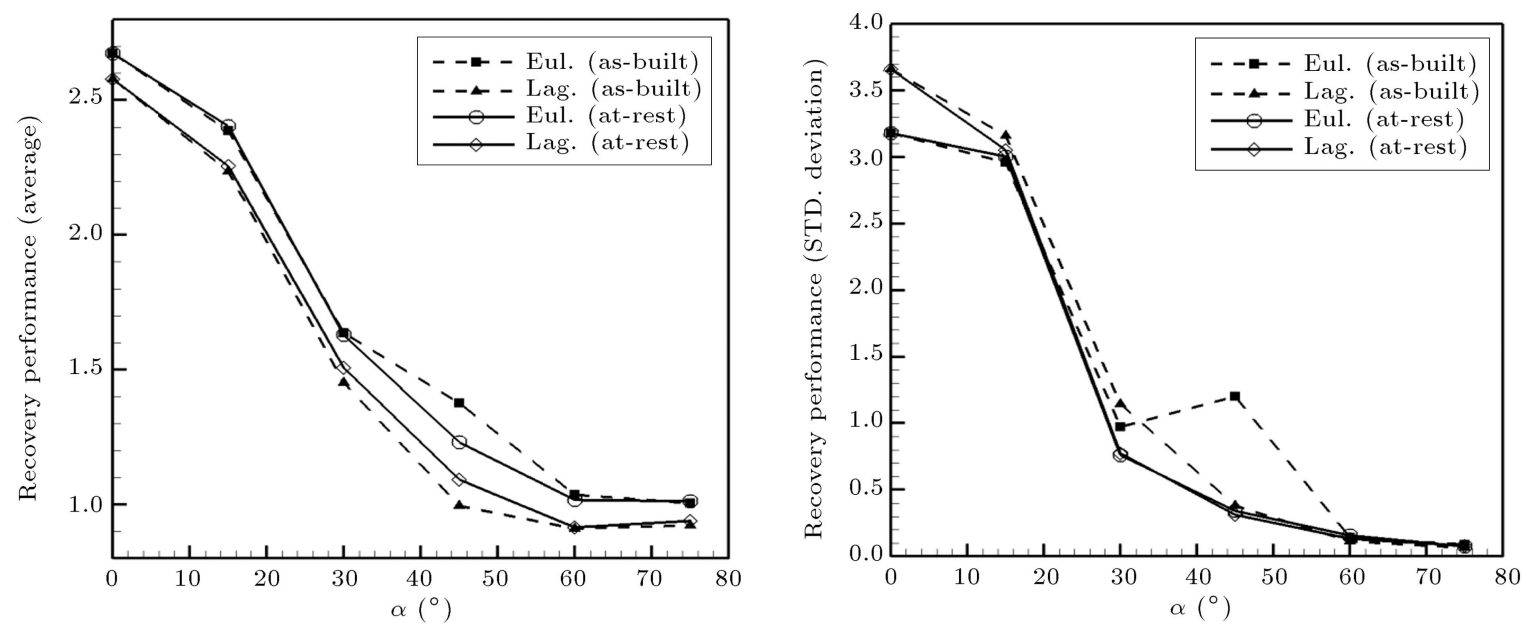

(b)
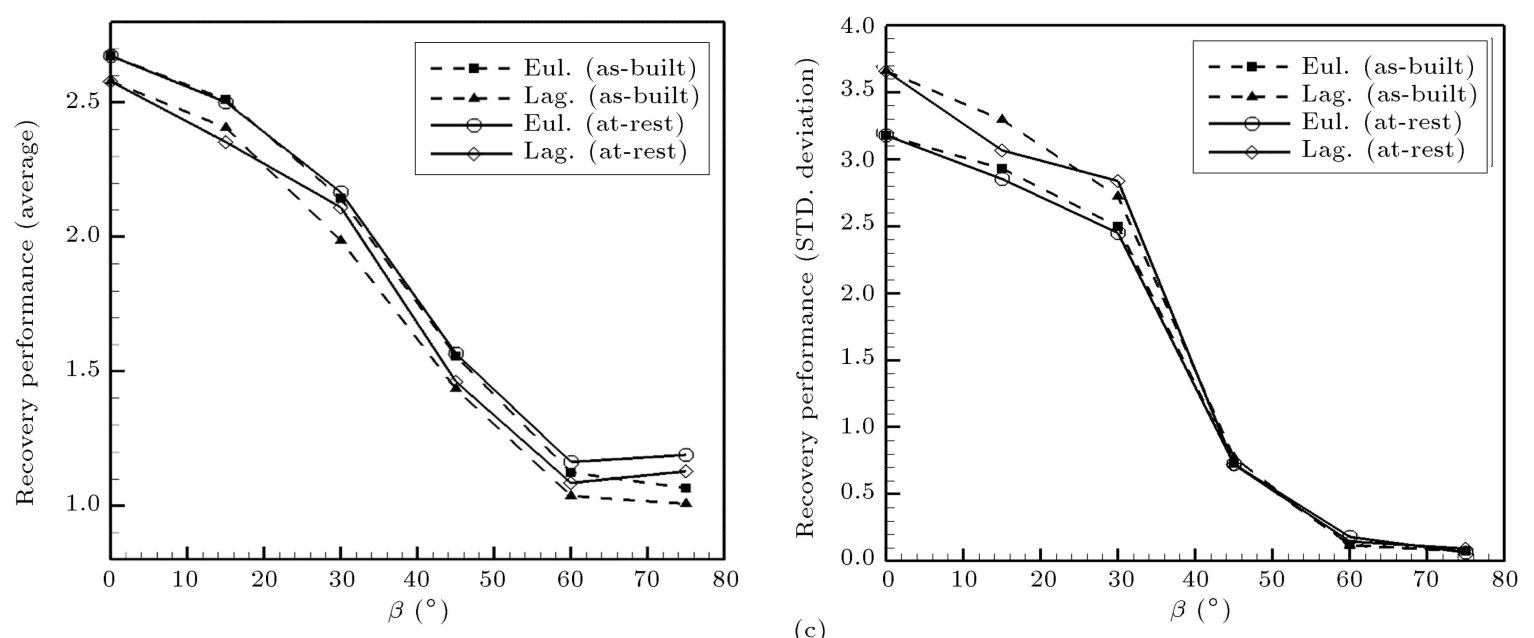

Figure 7. Change in average (left) and standard deviation (right) of recovery performance of all sampling units with air change rate (a), radial angle (b), and tangential angle (c).

Eulerian and Lagrangian analysis methods and both geometries, namely, as-built and at-rest, with variance in all three parameters. Covariance and standard deviation are calculated according to Eq. (38) for each case.
Results are shown in Figure 7. Specifically, geometry has little effect on both quantities. When air change rate is elevated, recovery performance calculation for sampling units shows that about half of these units during the entire or most of the recovery 
time are behind control volume analysis. By increasing ventilation rate, mean recovery performance increases, i.e., performance of ventilation increases in the entire room, which is a result of increased mixing and, naturally, a more turbulent field. However, standard deviation also follows a similar pattern, which shows the reason for increased overall performance, leading to enhancement of performance in a limited number of sampling units, not all of them.

A rise in radial angle reduces average and standard deviations, i.e., with increase in radial angle, mean value approaches 1 . Angle increase also has a declining effect on dispersion (except for one occasion), reaching about 0.1 at $75^{\circ}$. These diagrams show that radial pattern is significantly effective in keeping uniformity and homogeneity of the air flow inside the cleanroom.

Similar to the results obtained for radial angles, changes in tangential angle are accompanied by reduced mean and dispersion recovery performance; however, in comparison with radial angle, it demonstrates slightly worse performance in homogenizing room air.

\subsection{Minimum recovery performance and recovery time}

Importance of minimum recovery rate lies in the fact that it specifies the recovery time frame. Minimum recovery rate is shown in Figure 8 . In addition to analysis results and control volume interpretation, the first and sixth lowest recovery rate values calculated by Eulerian and Lagrangian analysis are shown. Lagrangian analysis results sometimes show erratic and even contradictory behavior.

Unlike Figure 4, in which control volume interpretation sometimes outpaces control volume analysis, in the first diagram, control volume interpretation is always behind, which is a result of curve fitting and has to be accepted to simplify the results.

The results show that increase in radial angle causes reduction in minimum efficiency, but leads to improvement of angles above 30 degrees in Lagrangian analysis and 45 degrees in Eulerian analysis. However, it never catches up control volume analysis while being higher than base state (0-degree angle) in some angles. Thus, although the mixing resulting from radial pattern is useful, it has little positive effect on minimum recovery efficiency.

As evident, in almost every scenario and in both approaches, recovery time decreases with growing ventilation rate. The general trend of change in recovery time is very similar to that in control volume analysis and interpretation, i.e., recovery time can be fitted to the function seen in Eq. (39).

Tangential angle has significant positive effects on minimum recovery efficiency. Increasing the angle to 15 degrees results in reduced minimum recovery efficiency; however, with further angle increase to 45 degrees, the value increases, surpassing even control volume analysis values. Then, with further increase, the value decreases, but it is still higher than that of the base state (0-degree angle). We also learn from results that change in ventilation rate cannot make a noticeable change in minimum recovery efficiency. A similar diagram for recovery time versus these three parameters is shown in Figure 7.

Therefore, it can be said that although ventilation rate has little influence on minimum recovery rate, none of the other parameters has a notable influence either. Thus, ventilation rate should be regarded as the most important quantity in recovery time determination.

By a more precise investigation into diagrams regarding variation in tangential and radial angles, it is clear that by mirroring the diagram regarding minimum recovery rate, horizontally, a diagram similar to that of recovery time can be obtained, that is, each minimum in a diagram in Figure 8 corresponds to a maximum in the equivalent diagram in the same figure. Results also show that the two approaches have high correlation, and recovery predictions of the Eulerian method are between $4 \%$ and $17 \%$ faster than those of the Lagrangian method.

An important conclusion from these diagrams is higher amount of this value in Eulerian analysis than in Lagrangian to an extent that the worst Eulerian results (rank 1) are comparable to the best Lagrangian results (rank 6). In addition, the best Eulerian analysis results (rank 6) are comparable to control volume interpretation results. Also, Eulerian analysis results follow a similar trend to that of control volume interpretation.

\section{Conclusion}

In the present work, a thorough investigation into recovery period was conducted. The air change rate and air inflow patterns were studied using the Eulerian and Lagrangian approaches as well as LES, DES, and $k-\omega$ SST turbulent models. Simulation results were evaluated against control volume analysis as well.

Results showed that $k-\omega$ SST predictions were not entirely consistent with experiments in both Eulerian and Lagrangian methods; but, compared to $k-\varepsilon$ RNG results, they were improved significantly. Besides, although LES and DES methods predicted concentration variations better in the Lagrangian method, Eulerian method provided better overall results.

According to the results, energy consumption and internal room pressure are directly proportional to rate of ventilation squared. Therefore, ventilation rate increase is suggested when it is going to replace traditional cleanroom pressurizing methods. Swirl diffusers can decrease energy consumption by reducing recovery time without any effect on mean room pressure; this can be significant in energy saving. 

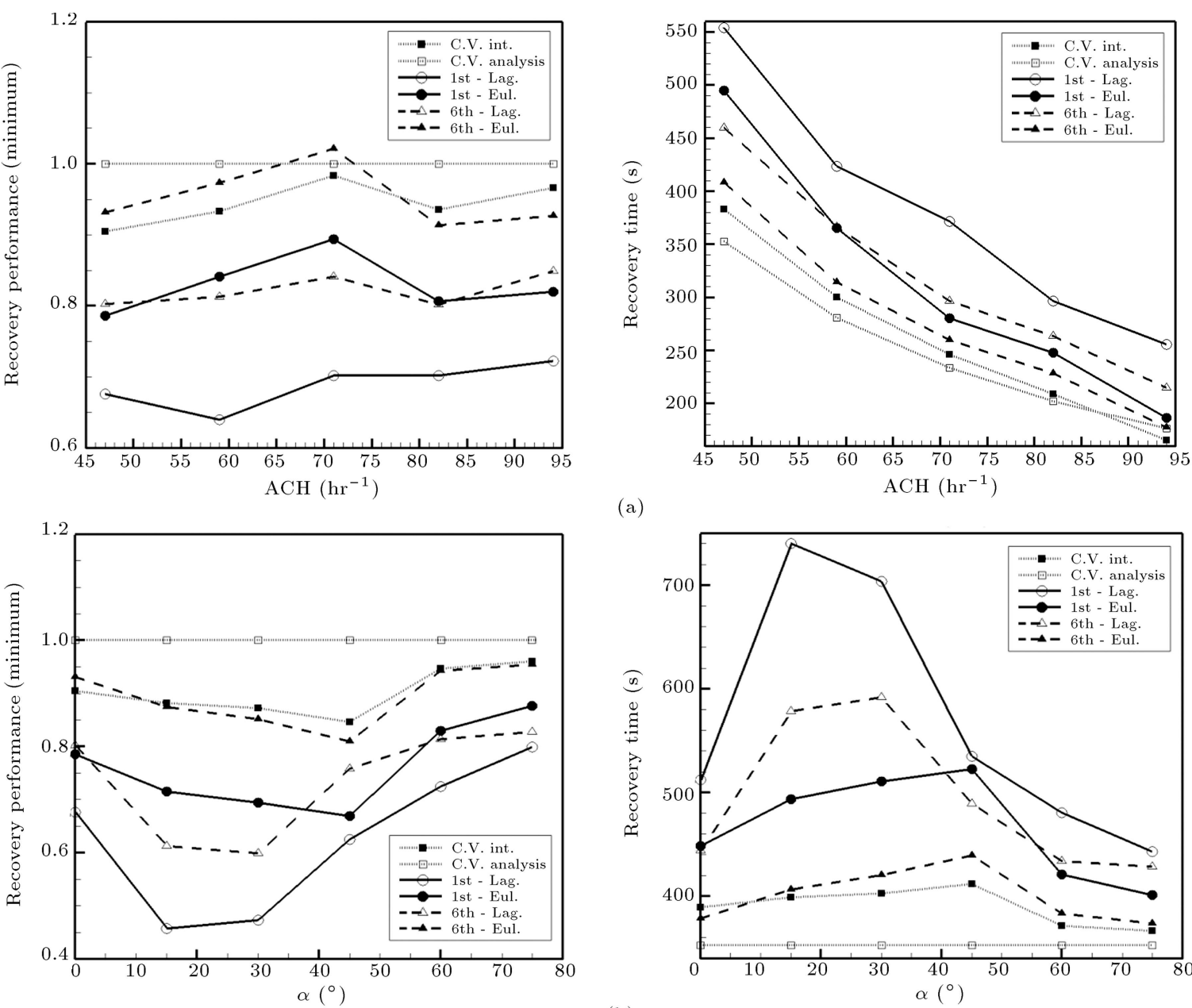

(a)
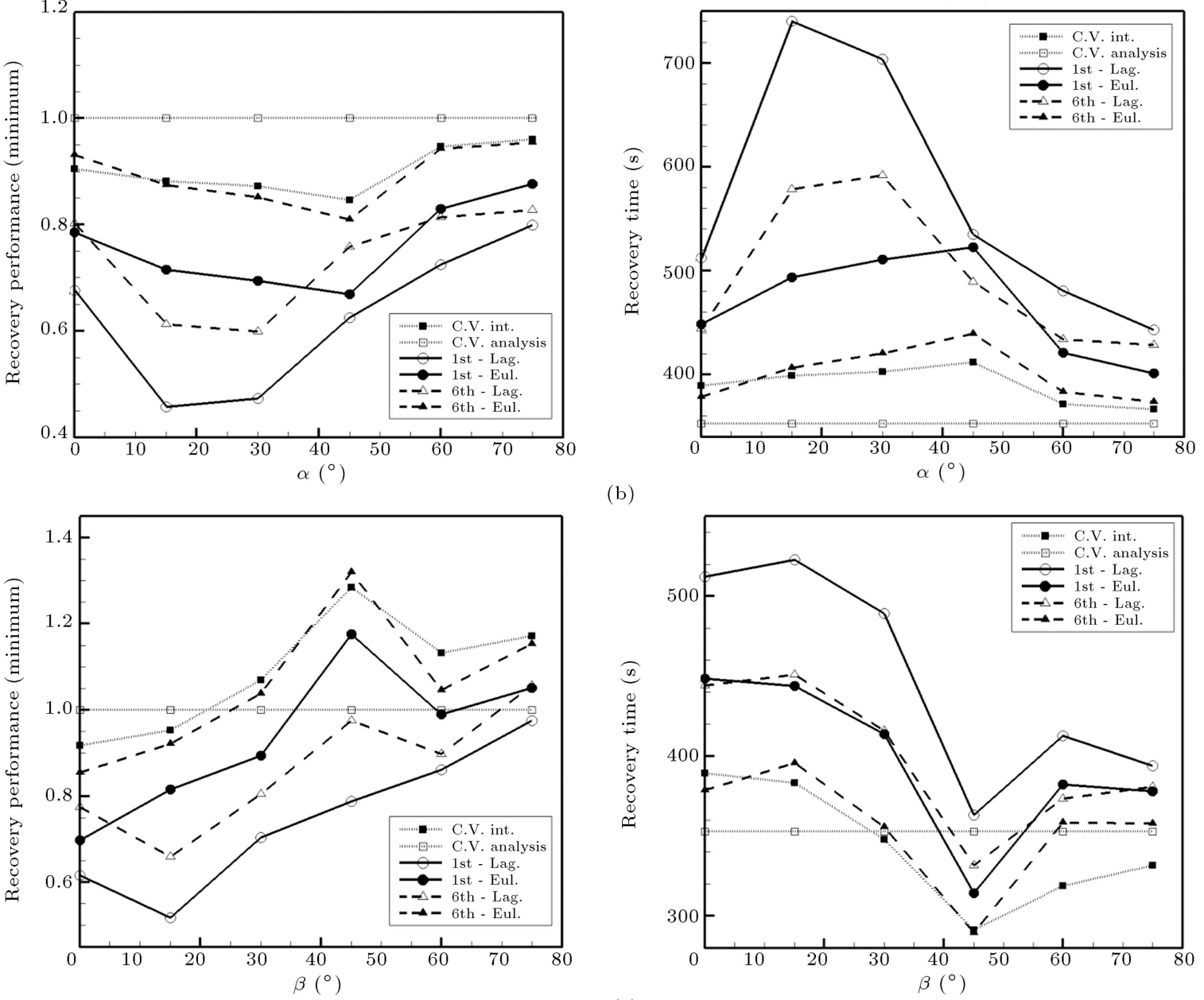

(b)

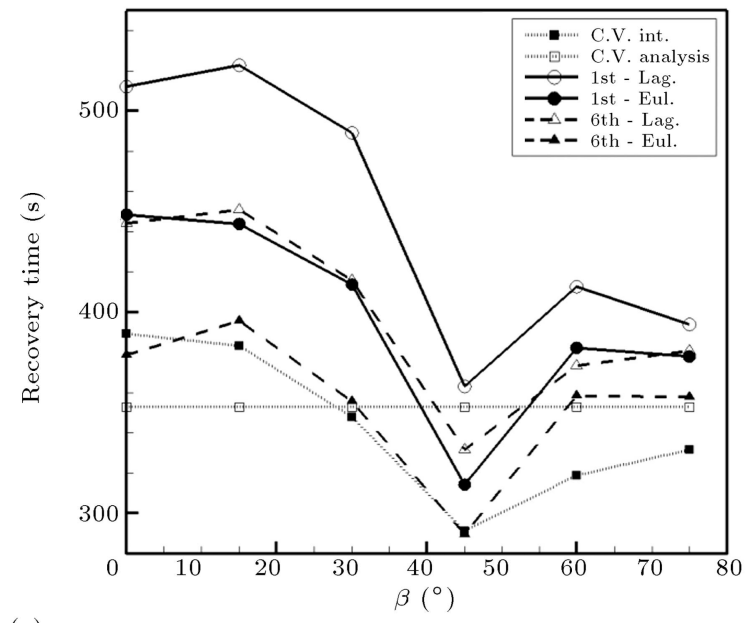

(c)

Figure 8. Change in minimum recovery performance (left) and recovery time deviation (right) with air change rate (a), radial angle (b), and tangential angle (c) in as-built mode.

Use of radial flow pattern in intake diffusers is significantly influential in homogenizing particle concentration; but it can increase the recovery time in some cases. Using the tangential (rotary) pattern of stream in intake diffusers greatly decreases recovery time while homogenizing particle concentration.
To study recovery in a cleanroom, sampling should be moved from below the intake diffusers closer to the exhaust vents. Presence of objects in the room has little effect on recovery.

In cleanroom recovery analysis, Eulerian and Lagrangian approaches present high correlation; but 
Eulerian method predicts a lower recovery time by $4 \%$ to $17 \%$.

\section{Nomenclature}

A Room area, $\mathrm{m}^{2}$

$A C H \quad$ Air Change rate, \#hr ${ }^{-1}$

$C \quad$ Species concentration, $\mathrm{kgm}^{-3}$

$C_{r} \quad$ Largest particles count, $\mathrm{m}^{-3}$

$C F \quad$ Correlation Factor, No Dim

COV Covariance, No Dim

$d \quad$ Diameter, $\mathrm{m}$

$D \quad$ Brownian diffusion, $\mathrm{m}^{2} \mathrm{~s}^{-1}$

F $\quad$ Force applied to particle, N

g Gravitational acceleration, $\mathrm{ms}^{-2}$

$k \quad$ Turbulent kinetic energy, $\mathrm{m}^{2}$

$l \quad$ Turbulent length scale, $\mathrm{m}$

$m \quad$ Mass, $\mathrm{kg}$

$N \quad$ Cleanliness level, No Dim

$N_{s} \quad$ Compulsory sampling units count, \#

$N_{S U} \quad$ Sampling unit number, No Dim

$p \quad$ Pressure $\mathrm{Pa}$

$Q \quad$ Inlet flow $\mathrm{m}^{3} \mathrm{~s}^{-1}$

$r \quad$ Random uniform number, No Dim

$S \quad$ Particle generation rate per unit of volume, $\# \mathrm{~m}^{-3}$

$\bar{S}_{i j} \quad$ Fluid strain rate, $\mathrm{s}^{-1}$

$u \quad$ Velocity $X$-component, $\mathrm{ms}^{-1}$

$\mathbf{u} \quad$ Velocity vector, $\mathrm{ms}^{-1}$

$\mathbf{u}_{r} \quad$ Particle-fluid relative velocity, $\mathrm{ms}^{-1}$

$v \quad$ Velocity $Y$-component, $\mathrm{ms}^{-1}$

$V \quad$ Volume, $\mathrm{m}^{3}$

$w \quad$ Velocity $Z$-component, $\mathrm{ms}^{-1}$

$W \quad$ Energy, J

$\alpha \quad$ Radial angle, deg.

$\beta \quad$ Tangential angle, deg.

$\delta_{i j} \quad$ Kronecker delta, No Dim

$\varepsilon \quad$ Turbulent energy dissipation rate, $\mathrm{m}^{2} \mathrm{~s}^{-3}$

$\eta_{f} \quad$ Filter efficiency, No Dim

$\mu \quad$ Fluid dynamic viscosity, $\mathrm{Nsm}^{-2}$

$\mu_{t} \quad$ Turbulent dynamic viscosity, $\mathrm{Nsm}^{-2}$

$\mu_{\text {eff }} \quad$ Fluid effective dynamic viscosity, $\mathrm{Nsm}^{-2}$

$v \quad$ Fluid Kinematic viscosity, $\mathrm{m}^{2} \mathrm{~s}^{-1}$

$\rho \quad$ Density, $\mathrm{kgm}^{-3}$

$\tau \quad$ Particle relaxation time, $\mathrm{s}$

$\begin{array}{ll}\tau_{S G S} & \text { Sub-grid scale stress in LES, } \mathrm{Nm}^{-2} \\ \nu & \text { Swirl diffuser outlet velocity, } \mathrm{ms}^{-1} \\ \omega & \begin{array}{l}\text { Turbulent energy specific dissipation } \\ \text { rate, } \mathrm{s}^{-1}\end{array} \\ \Psi & \text { Standard deviation, No Dim } \\ \zeta & \text { Gaussian random number, No Dim } \\ b & \text { Buoyancy } \\ f & \text { Fluid } \\ f r & \text { Drag } \\ g & \text { Gravity } \\ p & \text { Particle } \\ r & \text { Radial component } \\ z & \text { Axial component } \\ \theta & \text { Tangential component }\end{array}$

\section{References}

1. Lage, J., Bejan, A., and Anderson, R. "Efficiency of transient contaminant removal from a slot ventilated enclosure", International Journal of Heat and Mass Transfer, 34, pp. 2603-2615 (1991).

2. Lage, J., Bejan, A., and Anderson, R. "Removal of contaminant generated by a discrete source in a slot ventilated enclosure", International Journal of Heat and Mass Transfer, 35, pp. 1169-1180 (1992).

3. Mendez, C., San José, J., Villafruela, J., and Castro, F. "Optimization of a hospital room by means of CFD for more efficient ventilation", Energy and Buildings, 40, pp. 849-854 (2008).

4. Saidi, M., Sajadi, B., and Molaeimanesh, G. "The effect of source motion on contaminant distribution in the cleanrooms", Energy and Buildings, 43, pp. 966970 (2011).

5. Chen, S.-C., Tsai, C.-J., Li, S.-N., and Shih, H.-Y. "Dispersion of gas pollutant in a fan-filter-unit (FFU) cleanroom", Building and Environment, 42, pp. 19021912 (2007).

6. Khoo, C.Y., Lee, C.-C., and Hu, S.-C. "An experimental study on the influences of air change rate and free area ratio of raised-floor on cleanroom particle concentrations", Building and Environment, 48, pp. 84-88 (2012).

7. Wang, M., Lin, C.-H., and Chen, Q. "Advanced turbulence models for predicting particle transport in enclosed environments", Building and Environment, 47, pp. 40-49 (2012).

8. British Standard institution, BS EN ISO 14644, in Cleanrooms and Associated Controlled Environments - Part 2: Specifications for Testing and Monitoring to Prove Continued Compliance, Ed., UK (2000).

9. British Standard institution, BS EN ISO 14644, in Cleanrooms and Associated Controlled Environments - Part 1: Classification of Air Cleanliness, Ed., UK (1999). 
10. Chung, K.-C. and Wang, S.-K. "Study of appropriate indoor air quality models in the Taiwan area", Indoor and Built Environment, 3, pp. 149-155 (1994).

11. Sabatini, L., Analysis of Airborne Contamination Distribution in Mixed Flow Cleanroom and Consequences in the Control Techniques, Lesatec S.r.l. (2005).

12. Graebel, W., Advanced Fluid Mechanics, pp. 1-16, Academic Press (2007).

13. Rouaud, O. and Havet, M. "Computation of the air flow in a pilot scale clean room using $\mathrm{K}-\varepsilon$ turbulence models", International Journal of Refrigeration, 25(3), pp. 351-361 (2002).

14. Zhang, Z. and Chen, Q. "Comparison of the Eulerian and Lagrangian methods for predicting particle transport in enclosed spaces", Atmospheric Environment, 41, pp. 5236-5248 (2007).

15. Menter, F.R. "Review of the shear-stress transport turbulence model experience from an industrial perspective", International Journal of Computational Fluid Dynamics, 23, pp. 305-316 (2009).

16. Menter, F.R. "Two-equation eddy-viscosity turbulence models for engineering applications", AIAA journal, 32, pp. 1598-1605 (1994).

17. Fard, M.B. and Nikseresht, A. "Numerical simulation of unsteady 3D cavitating flows over axisymmetric cavitators", Scientia Iranica, 19, pp. 1258-1264 (2012).

18. Heidarinejad, G. "Turbulence" , pp. 3-163, Author, IR (2009).

19. Wilcox, D.C., Turbulence Modeling for CFD, pp. 63163, DCW industries La Canada, CA (1998).

20. Nikbakht, A., Abouali, O., and Ahmadi, G. "Nanoparticle beam focusing in aerodynamic lenses-an axisymmetric model", Scientia Iranica, 14, pp. 263-272 (2007).

21. Zhang, P., Roberts, R.M., and Bénard, A. "Computational guidelines and an empirical model for particle deposition in curved pipes using an Eulerian-
Lagrangian approach", Journal of Aerosol Science, 53, pp. 1-20 (2012).

22. Friedlander, S.K. and Smoke, D., Haze: Fundamentals of Aerosol Dynamics, Oxford University Press, US (2000).

23. Tominaga, Y. and Stathopoulos, T. "Turbulent Schmidt numbers for CFD analysis with various types of flowfield", Atmospheric Environment, 41, pp. 80918099 (2007).

24. Lu, W., Howarth, A.T., Adam, N., and Riffat, S.B. "Modelling and measurement of airflow and aerosol particle distribution in a ventilated two-zone chamber", Building and Environment, 31, pp. 417-423 (1996).

\section{Biographies}

Ali Pourfarzaneh received his BS degree from Isfahan University of Technology, Iran, and his MS degree from Tarbiat Modares University, Tehran, Iran, in Mechanical Engineering (Energy Conversion), in 2010 and 2014, respectively. His research interests include numerical modeling, computational fluid dynamics, and cleanroom.

Ali Jafarian received $\mathrm{BS}$, MS, and $\mathrm{PhD}$ degrees from Sharif University of Technology, Tehran, Iran, in 2000, 2002, and 2008, respectively, all in Mechanical Engineering (energy conversion). He is currently professor of Mechanical Engineering at Tarbiat Modares University, Tehran, Iran.

Hamidreza Kharinezhad Arani received his BS degree from Isfahan University of Technology, Iran, and his MS degree from Tarbiat Modares University, Tehran, Iran, in Mechanical Engineering (energy conversion), in 2014 and 2017, respectively. His research interests include computational fluid dynamics, numerical modeling, cleanroom, and turbulent dispersion of particles. 